\title{
Efficacy of Palliative Bladder Radiotherapy for Hematuria in Advanced Bladder Cancer Using Contemporary Radiotherapy Techniques
}

\author{
JEREMY TEY ${ }^{1}$, YU YANG SOON ${ }^{1}$, TIMOTHY CHEO $^{1}$, KIAT HUAT OOI ${ }^{1}$, FRANCIS HO ${ }^{1}$, \\ BALAMURUGAN VELLAYAPPAN ${ }^{1}$, DAVID CHIA ${ }^{1}$ and BEE CHOO TAI ${ }^{2}$ \\ ${ }^{1}$ Department of Radiation Oncology, National University Hospital, \\ National Cancer Institute of Singapore, Singapore, Singapore; \\ ${ }^{2}$ Saw Swee Hock School of Public Health, National University of Singapore, Singapore, Singapore
}

\begin{abstract}
Background/Aim: The aim of this study was to review the outcomes of palliative radiotherapy $(R T)$ for hematuria treated with modern RT techniques. Patients and Methods: This was a retrospective cohort study. The primary endpoint was symptom response rate. Secondary endpoints included symptom recurrence rate, overall survival and treatment-related toxicity. Results: Median age was 82 years (range $=36-98$ years). Median biologically effective dose (BED) was 36 Gy. Sixty-seven percent of patients (39/58) responded to $R T$. The median survival duration was 5.6 months (range $=0.02$ 47.6 months). One third (13/39) of responders had recurrence of hematuria. Competing Risk regression with death as the competing risk showed that patients treated with low BED regimen $(<36 \mathrm{~Gy})$ had 5.76 times the hazard of recurrence compared to high BED regimen (>36 Gy) $(p=0.01)$. One patient (2\%) developed grade 3 nausea and vomiting which required admission for intravenous hydration. Conclusion: BED regimens should be recommended as they are associated with a significantly lower rate of recurrent hematuria.
\end{abstract}

Bladder cancer ranks 7 th worldwide based on the age standardized incidence rate and accounted for approximately 550,000 new cases and 200,000 deaths worldwide in 2018 (1). Patients with bladder cancer often present with advanced disease. Patients with locally advanced disease with or

This article is freely accessible online.

Correspondence to: Dr. Jeremy Tey, MD, Radiation Oncologist, Department of Radiation Oncology, National University Hospital, National University Cancer Institute, NUHS Tower Block Level 7 , 1E Kent Ridge Road, 119228 Singapore, Singapore. Tel: +65 67724869, Fax: +65 67796320, e-mail: Jeremy_tey@nuhs.edu.sg

Key Words: Radiotherapy, bladder cancer, palliation, bleeding. without distant metastases often present with bleeding (hematuria). There are several treatment options for palliation of hematuria including palliative cystectomy, internal iliac artery embolization, bladder washout, transurethral resection of bladder tumour, cystodiathermy, intravesical formalin and palliative radiotherapy (RT).

Effective treatments with low toxicity are preferred for patients with advanced disease and limited life expectancy. Palliative RT has been shown to be effective in treating bleeding at other cancer sites, including lung and gastric cancers (2-5). It has the benefit of controlling the symptoms while reducing the burden of symptoms. There is limited evidence for the use of palliative RT in bladder cancer. Most studies report improvement of hematuria in $16-69 \%$ of patients (6-8). These studies had small patient numbers and used two-dimensional RT techniques for treatment. Currently, there is a limited number of reports on treatment toxicities for localized palliative bladder RT using modern RT techniques. In addition, there is currently no standard dose fractionation regimen for the palliation of hematuria. Palliative RT for hematuria can be delivered using high biologically effective dose (BED) regimens that typically entail daily treatments over weeks or more, or low BED regimens in which daily treatments are given over 5 days or less. At present, the response rates and duration of response of high versus low BED regimens in palliation of hematuria is not clear. If low BED regimens are equivalent to high BED regimens, then they should be the preferred treatment of choice due to increased convenience and shorter treatment time.

Therefore, the aim of this study was to review of the outcomes of palliative RT for bladder cancer at two tertiary hospitals in Singapore.

\section{Patients and Methods}

This study was a retrospective cohort study. The study was approved by the National Healthcare Group Institutional Review board. 
Patients. Eligibility criteria: The study included patients treated with palliative radiotherapy from January 2001 to December 2016. Patients aged 21 years and above with histopathological or radiological diagnosis of bladder cancer with hematuria, as the key index symptom, who received palliative intent radiotherapy were included. Tumour staging was based on the American Joint Cancer Committee (AJCC) 7th edition Cancer Staging Manual (9).

Exclusion criteria: Patients with previous abdominal/pelvic radiotherapy or those who received concurrent chemotherapy were excluded.

Data collection. Data was obtained from the electronic medical records of the National University Hospital and the Tan Tock Seng Hospital. Index symptoms were identified. Data collected included patient demographics such as gender, age, smoking status and Eastern Cooperative Oncology Group (ECOG) performance status. Tumour details collected included tumour stage, histology and grade. Radiotherapy details collected included radiotherapy dose fractionation regimens, total radiotherapy dose, treatment technique and whether Computed Tomography (CT) planning was used. Details of chemotherapy used before or after RT were collected.

Covariates. Covariates studied included BED ( $<36 \mathrm{~Gy} v s . \geq 36 \mathrm{~Gy})$, age $(<70 v s . \geq 70)$, gender (male $v s$. female), smoking (non-smoker $v s$. current smokers, ex-smokers, unknown), ECOG performance status (2, 3, 4 vs. 1), stage (locally advanced vs. metastatic) and bleeding response (Yes vs. No).

Outcomes. Primary outcome: The primary outcome was response rate for hematuria after palliative RT to the bladder. Response was defined as no further blood transfusion needed after RT and within one week of completion of RT and no further hematuria following RT and within one week of completion of RT.

Secondary outcomes: The following were the secondary outcomes considered and their respective definitions:

1. Duration of bleeding palliation was defined as the time from response of patients who achieved satisfactory palliation until recurrence of hematuria or death.

2. To quantify the percent net bleeding symptom relief, the duration of relief from hematuria and survival time from RT was first determined for each patient. The ratio from duration of relief from hematuria and survival multiplied by 100 was calculated to obtain the "percent net symptom relief". This represented the percentage of the remaining patient's life after RT that was spent with relief of hematuria and without need for further treatment (10).

3 . Bleeding recurrence was defined as recurrence of hematuria after initial response.

4.Overall survival time was defined as survival from the end of radiotherapy to death from all causes.

5. Treatment toxicity would be retrospectively scored using the Common Toxicity Criteria (CTC) v4.0 (11)

Follow-up. Patients were followed up 1 month after the completion of RT and thereafter every 3 months with physical examination. Data was censored at December 2016. Laboratory investigations were performed at the discretion of the treating radiation oncologist.

Statistical analysis. Frequency with percentage and median with range (minimum-maximum) were used to describe the patient and tumour characteristics of this study cohort for categorical and
Table I. Patient and tumour characteristics.

\begin{tabular}{|c|c|c|c|c|}
\hline $\begin{array}{l}\text { Patient } \\
\text { characteristics }\end{array}$ & $\begin{array}{l}\text { Total } \\
(\mathrm{n}=58)\end{array}$ & $\begin{array}{l}\text { Low BED } \\
(\mathrm{n}=36)\end{array}$ & $\begin{array}{l}\text { High BED } \\
\quad(\mathrm{n}=22)\end{array}$ & $p$-Value \\
\hline \multicolumn{4}{|l|}{ Age (years) $(\%)$} & 0.02 \\
\hline$\geq 70$ & $46(79)$ & $32(89)$ & $14(64)$ & \\
\hline$<70$ & $12(21)$ & $4(11)$ & $8(36)$ & \\
\hline \multicolumn{4}{|l|}{ Gender (\%) } & 0.97 \\
\hline Male & $42(72)$ & $26(72)$ & $16(28)$ & \\
\hline Female & $16(28)$ & $10(28)$ & $6(72)$ & \\
\hline \multicolumn{4}{|l|}{ Smoking (\%) } & 0.65 \\
\hline Never & $21(36)$ & $13(36)$ & $8(37)$ & \\
\hline Current & $7(12)$ & $3(8)$ & $4(18)$ & \\
\hline Ex-smoker & $10(17)$ & $6(17)$ & $4(18)$ & \\
\hline Unknown & $20(35)$ & $14(39)$ & $6(27)$ & \\
\hline \multicolumn{5}{|l|}{ Performance status } \\
\hline$(\mathrm{ECOG})(\%)$ & & & & 0.11 \\
\hline 1 & $23(40)$ & $11(30)$ & $12(54)$ & \\
\hline 2 & $10(17)$ & $5(14)$ & $5(23)$ & \\
\hline 3 & $17(29)$ & $14(39)$ & $3(14)$ & \\
\hline 4 & $8(14)$ & $6(17)$ & $2(9)$ & \\
\hline \multicolumn{4}{|l|}{ Race $(\%)$} & 0.59 \\
\hline Chinese & $45(77)$ & $28(78)$ & $17(77)$ & \\
\hline Indian & $8(14)$ & $5(14)$ & $3(13)$ & \\
\hline Malay & $4(7)$ & $3(8)$ & $1(5)$ & \\
\hline Others & $1(2)$ & $0(0)$ & $1(5)$ & \\
\hline \multicolumn{5}{|l|}{ Tumour characteristics } \\
\hline \multicolumn{4}{|l|}{ T-stage $(\%)$} & 0.45 \\
\hline $\mathrm{T} 1$ & $1(2)$ & $0(0)$ & $1(5)$ & \\
\hline $\mathrm{T} 2$ & $17(29)$ & $12(33)$ & $5(23)$ & \\
\hline $\mathrm{T} 3$ & $22(38)$ & $14(39)$ & $8(36)$ & \\
\hline $\mathrm{T} 4$ & $17(29)$ & $9(25)$ & $8(36)$ & \\
\hline Unknown & $1(2)$ & $1(3)$ & $0(0)$ & \\
\hline \multicolumn{4}{|l|}{$\mathrm{N}$-stage $(\%)$} & 0.21 \\
\hline 0 & $44(76)$ & $27(75)$ & $17(77)$ & \\
\hline 1 & $2(3)$ & $1(3)$ & $1(5)$ & \\
\hline 2 & $4(7)$ & $4(10)$ & $0(0)$ & \\
\hline 3 & $6(11)$ & $2(6)$ & $4(18)$ & \\
\hline $\mathrm{x}$ & $2(3)$ & $2(6)$ & $0(0)$ & \\
\hline \multicolumn{4}{|l|}{ Stage (\%) } & 0.49 \\
\hline Locally advanced & $40(69)$ & $26(72)$ & $14(64)$ & \\
\hline Metastatic & $18(31)$ & $19(28)$ & $8(36)$ & \\
\hline \multicolumn{4}{|l|}{ Histology (\%) } & 0.32 \\
\hline $\mathrm{TCC}$ & $47(81)$ & $27(75)$ & $20(91)$ & \\
\hline Unknown & $11(19)$ & $9(25)$ & $2(9)$ & \\
\hline \multicolumn{4}{|l|}{ Tumour grade $(\%)$} & 0.33 \\
\hline 1 & $0(0)$ & $0(0)$ & $0(0)$ & \\
\hline 2 & $1(2)$ & $0(0)$ & $1(5)$ & \\
\hline 3 & $49(84)$ & $30(83)$ & $19(86)$ & \\
\hline Unknown & $8(14)$ & $6(17)$ & $2(9)$ & \\
\hline
\end{tabular}

ECOG: Eastern Cooperative Oncology Group; BED: biologically effective dose; T: tumour stage; N: nodal stage; TCC: transitional cell carcinoma.

continuous variables respectively. For the primary outcome, the proportion of patients experiencing symptom relief was reported. Logistic regression was performed to identify factors associated with bleeding response. Secondary outcome on duration of symptom response and percent net relief were reported descriptively as 
Table II. Bleeding response and recurrence according to BED.

\begin{tabular}{|c|c|c|c|c|c|c|}
\hline & Low BED & $\%$ & High BED & $\%$ & Total & $\%$ \\
\hline Bleeding response & $\mathrm{N}=36$ & & $\mathrm{~N}=22$ & & $\mathrm{~N}=58$ & \\
\hline No & 14 & 39 & 5 & 13 & 19 & 33 \\
\hline Yes & 22 & 61 & 17 & 77 & 39 & 67 \\
\hline Bleeding recurrence & $\mathrm{N}=22$ & $\%$ & $\mathrm{~N}=17$ & $\%$ & $\mathrm{~N}=39$ & $\%$ \\
\hline No & 11 & 50 & 15 & 87 & 26 & 67 \\
\hline Yes & 11 & 50 & 2 & 13 & 13 & 33 \\
\hline
\end{tabular}

BED: Biologically effective dose.

median and mean, respectively. Differences in response and recurrence rates between high BED (RT regimen with BED above or equal to the median BED) and low BED (RT regimen with BED below the median BED) regimens using the median BED as the cut off were compared. Overall survival was described using the Kaplan-Meier curves with survivors censored on the last date known to be alive. Difference in survival curves between high and low BED was compared using the log rank test. Cox proportional hazard model was performed to identify independent factors associated with survival. Competing risk regression with death as a competing risk, using the backward elimination method was performed to identify variables associated with symptom recurrence (12). For adverse events outcomes, the proportion of patients experiencing a grade 3 or above adverse events was reported. Statistical analysis was performed using STATA v14. A two-sided $p$-value of $<0.05$ was considered to indicate statistical significance.

\section{Results}

Baseline characteristics of study the population. From January 2001 to December 2016, a total of 58 patients with bladder cancer underwent palliative RT for hematuria. Median follow-up was 24.3 months (range=0-47.6 months) months. Median age was 82 years (range $=36-98$ years) years (Table I). Significantly more patients aged 70 years and above received low BED regimen as compared to high $\mathrm{BED}$ regimen. Approximately $90 \%$ of patients in the low BED group were aged 70 years or older, as compared to $64 \%$ in the high BED group. The majority of patients were male (42/58), and Chinese (45/58). Regarding smoking, 21 (36\%) patients were never smokers, $7(12 \%)$ were smokers, 10 $(17 \%)$ were ex-smokers and smoking history was not available in $20(35 \%)$ patients. Forty-seven $(81 \%)$ patients had transitional cell carcinoma (TCC) (Table I). Eighteen (31\%) patients had T1-2 disease and $39(67 \%)$ had T3-4 disease. Eighteen (31\%) patients had known distant metastatic disease at presentation. The majority of the patients $(84 \%)$ had grade 3 tumours.

Pelvic radiotherapy treatment details. The RT dose fractionation regimens were heterogeneous, ranging from $8 \mathrm{~Gy}$ in a single fraction to $40 \mathrm{~Gy}$ in 16 fractions. The median BED was 36 Gy. Thirty $(62 \%)$ patients received a BED of $<36$ Gy (low BED) and 22(38\%) received a BED of $\geq 36$ Gy (high BED). Fifty-three of 58 patients (91\%) were CT planned. The distribution of patients who were treated with two, three and four field plans were 20/58 (35\%), 23/58 $(40 \%)$ and $15 / 58(25 \%)$, respectively.

Palliative chemotherapy treatment details. Five patients received palliative chemotherapy. Two patients received gemcitabine/cisplatinum chemotherapy prior to RT, two patients received gemcitabine/carboplatin chemotherapy (one patient before RT and one patient after RT) and one patient received intravesical mitomycin-C prior to $\mathrm{RT}$.

Recurrent hematuria treatment details. Patients who recurred were treated with bladder washout, re-RT, blood transfusion, cystodiathermy or fluid resuscitation. Thirteen patients had recurrence hematuria. All thirteen patients responded to the treatment for recurrent hematuria. Six patients received reRT for recurrent hematuria. One patient received $6 \mathrm{~Gy}$ in a single fraction, 2 patients received 20 Gy in 10 fractions, one patient received $30 \mathrm{~Gy}$ in 12 fractions, one patient received $30 \mathrm{~Gy}$ in 10 fractions and one patient received $20 \mathrm{~Gy}$ in 8 fractions.

Primary outcome: Symptom response. Thirty-nine (67\%) patients responded to palliative RT. Twenty-two of 36 patients $(61 \%)$ who were treated with low BED regimen responded while 17 of $22(77 \%)$ patients who were treated with high BED regimen responded. The median duration of response was 3.7 months (range $=0-44.5$ months). Mean percent net relief was $86.9 \%$ (range $=10-100 \%$ ). Table II details bleeding response and recurrence according to BED. Factors including BED, age, gender, smoking, performance status and stage were not found to be significantly associated with bleeding response (Table III).

Secondary outcome: Symptom recurrence. Thirteen of 39 (33\%) patients developed recurrence of hematuria. Eleven of $22(50 \%)$ patients who were treated with low BED regimen 


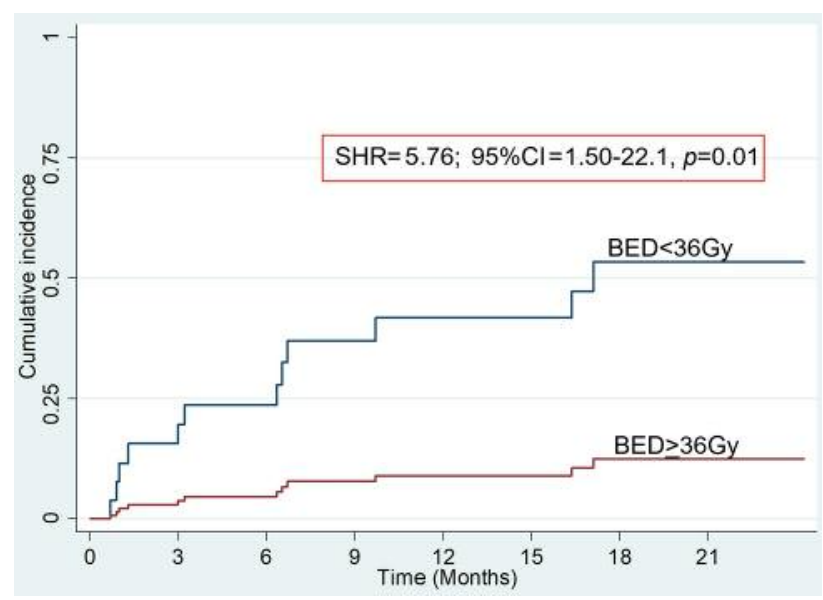

Figure 1. Cumulative incidence of recurrence of hematuria according to $B E D$.

Table III. Multivariate analysis of factors associated with hematuria response.

\begin{tabular}{lccc}
\hline Bleeding response & OR & $95 \% \mathrm{CI}$ & $p$-Value \\
\hline BED & & & \\
$\quad \geq 36$ Gy & 1.00 & & \\
$\quad<36 \mathrm{~Gy}$ & 1.51 & $0.37-6.21$ & 0.57 \\
Age & & & \\
$\quad<70$ & 1.00 & & \\
$\quad \geq 70$ & 0.14 & $0.15-1.43$ & 0.10 \\
Gender & & & \\
$\quad$ Male & 1.00 & & \\
Female & 2.86 & $0.57-14.3$ & 0.20 \\
Smoking & & & \\
$\quad$ Non-smoker & 1.00 & & \\
$\quad$ Current & 1.99 & $0.22-17.8$ & 0.54 \\
Ex-smoker & 0.72 & $0.10-5.0$ & 0.74 \\
$\quad$ Unknown & 1.20 & $0.27-5.33$ & 0.81 \\
Performance status & & & \\
1 & 1.00 & & \\
2 & 0.17 & $0.02-1.17$ & 0.07 \\
3 & 0.42 & $0.08-2.22$ & 0.31 \\
4 & 0.89 & $0.11-6.95$ & 0.90 \\
Stage & & & \\
$\quad$ Locally advanced & 1.00 & & \\
Metastatic & 0.91 & $0.23-3.62$ & 0.99 \\
\hline
\end{tabular}

BED: Biologically effective dose.

developed recurrent hematuria while 2 of 17 (13\%) patients who were treated with high BED regimen developed recurrent hematuria. Competing risk regression (with death as competing risk) showed that the hazard of recurrent hematuria was increased in low BED regimen (Subdistribution HR=5.75;

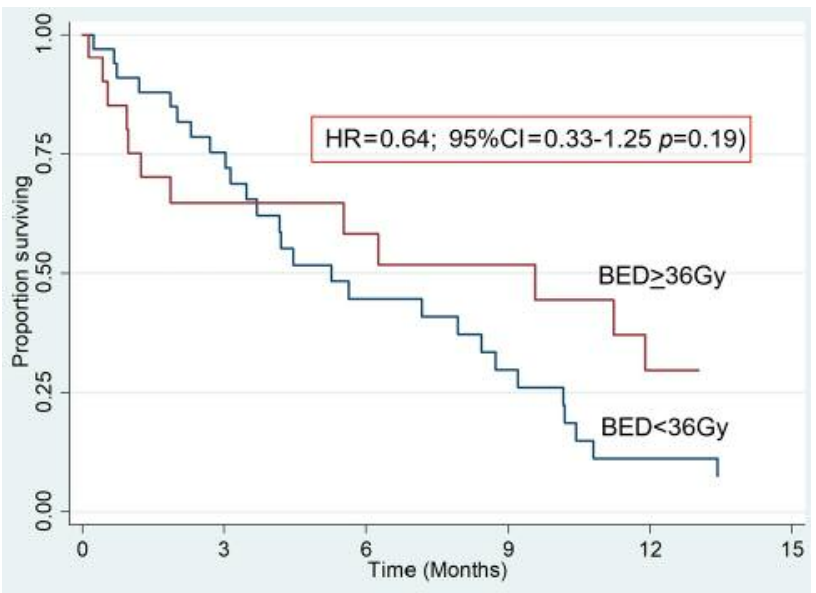

Figure 2. Overall survival according to BED.

Table IV. Factors significantly associated with recurrent hematuria.

\begin{tabular}{lccc}
\hline Bleeding recurrence & SHR & $95 \% \mathrm{CI}$ & $p$-Value \\
\hline BED & & & \\
$\quad>36 \mathrm{~Gy}$ & 1.00 & & \\
$\quad<36 \mathrm{~Gy}$ & 5.76 & $1.50-22.1$ & 0.01 \\
Smoking & & & 0.11 \\
$\quad$ Non-smoker & 1.00 & $1.10-17.31$ & \\
Current & 4.36 & $0.07-7.40$ & 0.04 \\
Ex-smoker & 0.74 & $0.35-6.95$ & 0.80 \\
Unknown & 1.56 & & 0.56 \\
\hline
\end{tabular}

BED: Biologically effective dose; SHR: subdistribution hazard ratio.

95\% $\mathrm{CI}=1.50-2.21, p=0.01)$ as compared to high BED regimen, and in current smokers $(\mathrm{SHR}=4.36 ; 95 \% \mathrm{CI}=1.10$ 17.31, $p=0.04$ ) as compared to nonsmokers (Figure 1) (Table IV). Factors such as age, gender, performance status and stage were not significantly associated with the risk of recurrent hematuria.

Duration of response and percent net relief. Median duration of response was 3.7 (range $=0-44.5$ ) months. Percent net relief was $86.9 \%$ (range $=10-100 \%$ ).

Survival. The median survival of all patients was 5.6 months (range $=0-47.6$ months). Median survival of responders was 5.6 months (range $=0-47.6$ months) as compared to 3.5 months (range $=0-13.4$ months) of non-responders. The 30day mortality was $14 \%(n=8)$. Cox regression analysis suggested that patients with ECOG performance status 4 had 
7.35 times the hazard of death compared to patients with ECOG performance $1 \quad(\mathrm{HR}=7.35 ; 95 \% \mathrm{CI}=2.13-23.40$, $p<0.01)$. BED was not significantly associated with mortality $(\mathrm{HR}=0.64 ; 95 \% \mathrm{CI}=0.33-1.25 p=0.19)$ (Figure 2). In addition, factors such as age, gender, smoking, stage and response of hematuria to palliative RT were not significantly associated with increased hazard of mortality.

Treatment toxicities. Acute toxicity: Palliative RT was well tolerated. Two patients $(3.5 \%)$ had grade 1 or grade 2 diarrhea. One patient had grade 1 pelvic pain. Another patient $(1.7 \%)$ had grade 3 nausea and vomiting requiring admission for intravenous hydration.

Late toxicity: No grade 3 or higher late toxicities were recorded.

\section{Discussion}

This study showed that palliative RT for hematuria in patients with bladder cancer was associated with high response rates. There was no significant difference in response rates of hematuria between high BED and low BED regimens. Patients treated with low BED regimen had a significantly increased hazard for recurrence of hematuria. Treatment was also well tolerated with only one grade 3 acute toxicity recorded $(2 \%)$.

Our results are consistent with outcomes of palliative radiotherapy for other tumour sites $(2,3,5)$. In a systematic review of palliative RT for hemoptysis in lung cancer, Fairchild et al. have reported that there was no significant difference in response rates between high dose versus low dose regimens (2). Similarly, a systematic review of palliative radiotherapy for gastric cancer did not find a significant difference in response rates of gastric cancer bleeding between patients treated with high BED versus low BED regimens (3). A retrospective review, however, found a trend for poorer control in patients with gastric cancer treated with low BED regimens (13). In this study, we found no factor significantly associated with bleeding response.

Patients who responded to RT had a durable response. This was reflected in the mean percent net relief. The mean percent net relief was $86.9 \%$ which indicated that on average, patients were free from hematuria for $86.9 \%$ of their remaining life. In addition, the median duration of response is 3.7 months, which is close to the median survival of 5.6 months. Although there was no difference in initial bleeding response between low and high BED regimens, we found that patients treated with low BED regimen had a significantly increased hazard for recurrence of hematuria. For patients who had responded to RT, death was regarded a competing risk for recurrence of hematuria as these patients had advanced disease with limited life expectancy. Competing risk regression with death as a competing risk showed that patients treated with high BED regimens were significantly less likely to have recurrent hematuria. This finding is important. Many radiation oncologists prescribe low BED regimens for patients with hematuria due to shorter treatment time, increased patient convenience and possibly lesser side effects. Our study suggests that high BED regimens should be prescribed whenever possible to prevent recurrent hematuria which may lead to recurrent hospitalizations, increased hospital stay and increased costs in the long term. In addition, patients are at risk for side effects of retreatment of recurrent hematuria such as radiation cystitis. The benefit of high $B E D$ regimens in producing a more durable response compared to low BED regimens is also acknowledged in trials of palliative RT for lung cancer (2).

We found that current smokers have increased hazard for recurrent hematuria compared to nonsmokers. Current smokers have a worse prognosis compared to non-smokers and there is a strong biological basis underlying this finding. The biological effect of RT is mediated via the production of free radicals, a process that is enhanced in the presence of oxygen. Well oxygenated cells are two to three times more radiosensitive then hypoxic cells. Cigarette smoking produces chronic hypoxia via chronic exposure to carbon monoxide which binds to haemoglobin and decreases oxygen availability (14). The detrimental effects of smoking during RT have also been seen in other tumour sites. In a matched case control study of 202 patients with squamous cell carcinoma of the head and neck, it was found that patients who smoked during RT had significantly worse 5-year overall survival, locoregional control and overall survival (15). Similarly in prostate cancer, a systematic review of the association of smoking status among patients undergoing radiotherapy or surgery has found that current smokers were at higher risk of developing biochemical recurrence, metastases and cancer specific mortality as compared to nonsmokers (16).

The ECOG performance scale (17) was developed to assess how a disease is affecting the activities of daily living of the patient and therefore aids in determining treatment and management. Studies have shown that for patients with advanced cancer, survival was approximately halved for each deterioration of performance level based on the ECOG performance score (18). Similarly, our study showed that increased ECOG levels were associated with worse prognosis. Patients with ECOG performance status of four had 7.35 times the hazard of death compared to patients with ECOG performance status of one.

Treatment was well tolerated with one patient having grade 3 nausea/vomiting. This may be because of the improvements in RT planning and delivery. Prior studies of palliative bladder radiotherapy included RT treatment based on 2-dimensional (2D) simulation and Anterior-posterior 
(AP)/Posterior-Anterior (PA) field arrangements. Ninety-one percent of patients were treated with 3DCRT, and more than $60 \%$ of patients were treated with a multifield plan. This may have accounted for the low toxicity rates.

The strengths of our study are that first, we performed competing risk analysis with death as a competing risk for recurrence of hematuria outcome instead of the Cox proportional hazard model. It has been shown that when the Cox proportional hazard model is used to describe outcomes other than all-cause mortality, the results may be biased if there is a related competing risk such as death (19-21). Second, the response rates in our study are consistent with the published literature. Third, there is no randomized trial data providing evidence regarding the incidence of recurrent hematuria amongst patients treated with high versus low BED. As far as we know, this is the first study that compared hematuria recurrence rates among patients who had an initial response to low and high BED regimens. Lastly, we provided toxicity outcomes of patients treated with modern radiotherapy techniques. We showed that palliative bladder $\mathrm{RT}$ is well tolerated in the modern era (i.e. incidence of grade 3 or greater nausea/vomiting is $<2 \%$ ), with the dose fractionation regimens prescribed in this study.

There are several limitations to our study. First, the small sample size might not provide enough power to demonstrate differences in response rates and survival between low and high BED regimens. Second, this is a retrospective study which might be prone to reviewer bias, leading to underreporting treatment toxicity. Third, being a nonrandomized study, there may be confounders that were not recognized and hence they were not adjusted for.

The implications of this study are that a high BED radiotherapy regimen should be prescribed whenever possible for palliation of hematuria from bladder cancer. This is because a higher BED regimen is associated with a significantly lower rate of recurrent hematuria. This may translate to decreased readmissions for treatment of recurrent bleeding, improving the patient's quality of life. Toxicities of retreatment can be avoided. In addition, it may also be costeffective due to avoidance of retreatment and hospital stay.

\section{Conclusion}

Palliative bladder RT is associated with high response rates irrespective of low or high BED regimens. High BED regimens should be prescribed whenever possible. Future randomized trials incorporating cost and quality of life analysis should be performed to confirm the benefit of high versus low BED regimens.

\section{Conflicts of Interest}

The Authors declare no conflicts of interest with regard to this study.

\section{Authors' Contributions}

JT: formulated the protocol, data collection, data analysis, interpretation of data, manuscript writing; YYS: Interpretation of data, revision of manuscript; TC: Interpretation of data, revision of manuscript; KHO: Interpretation of data, revision of manuscript; FH: Interpretation of data, revision of manuscript; BV: Interpretation of data, revision of manuscript; DC: Interpretation of data, revision of manuscript; BCT: Formulated the protocol, interpretation of data, revision of manuscript.

\section{References}

1 Bray F, Ferlay J, Soerjomataram I, Siegel RL, Torre LA and Jemal A: Torre, Ahmedin Jemal. Global Cancer Statistics 2018: Globocan estimates of incidence and mortality worldwide for 36 cancers in 185 countries. CA Cancer J Clin 68(6): 394-424, 2018. PMID 30207593. DOI: 10.3322/caac.21492

2 Fairchild A, Harris K, Barnes E, Wong R, Lutz S, Bezjak A, Cheung $P$ and Chow E: Palliative thoracic radiotherapy for lung cancer: A systematic review. J Clin Oncol 26(24): 4001-4011, 2008. PMID: 18711191 . DOI: $10.1200 /$ JCO.2007.15.3312

3 Tey J, Soon YY, Koh WY, Leong CN, Choo BA, Ho F, Vellayappan B, Lim K and Tham IW: Palliative radiotherapy for gastric cancer: A systematic review and meta-analysis. Oncotarget 8(15): 25797-25805, 2017. PMID: 28445941. DOI: 10.18632/oncotarget.15554

4 Tey J, Zheng H, Soon YY, Leong CN, Koh WY, Lim K, So JBY, Shabbir A, Tham IWK and Lu J: Palliative radiotherapy in symptomatic locally advanced gastric cancer: A phase ii trial. Cancer Med 8(4): 1447-1458, 2019. PMID 30790469. DOI: 10.1002/cam4.2021

5 Chia D, Lu J, Zheng H, Loy E, Lim K, Leong C, Wong L, Tan G, Chen D, Ho F and Tey J: Efficacy of palliative radiation therapy for symptomatic rectal cancer. Radiother Oncol 121(2): 258-261, 2016. PMID: 27745911. DOI: 10.1016/i.radonc.2016.06.023

6 Duchesne GM, Bolger JJ, Griffiths GO, Trevor Roberts J, Graham JD, Hoskin PJ, Fossa SD, Uscinska BM and Parmar MK: A randomized trial of hypofractionated schedules of palliative radiotherapy in the management of bladder carcinoma: Results of medical research council trial ba09. Int J Radiat Oncol Biol Phys 47(2): 379-388, 2000. PMID: 10802363. DOI: 10.1016/s0360-3016(00)00430-2

7 Lacarriere E, Smaali C, Benyoucef A, Pfister C and Grise P: The efficacy of hemostatic radiotherapy for bladder cancer-related hematuria in patients unfit for surgery. Int Braz J Urol 39(6): 808-816, 2013. PMID: 24456773. DOI: 10.1590/S16775538.IBJU.2013.06.06

8 Srinivasan V, Brown $\mathrm{CH}$ and Turner AG: A comparison of two radiotherapy regimens for the treatment of symptoms from advanced bladder cancer. Clin Oncol (R Coll Radiol) 6(1): 1113, 1994. PMID: 7513538.

9 AJCC: Cancer Staging Manual Seventh Edition. The American Joint Cancer Committee, Chicago, IL, USA, 2010. Available from: https://cancerstaging.org/references-tools/deskreferences/ Documents/AJCC\%207th\%20Ed\%20Cancer\%20Staging\%20Manu al.pdf (last accessed September 10, 2019)

10 Salazar OM, Rubin P, Hendrickson FR, Komaki R, Poulter C, Newall J, Asbell SO, Mohiuddin M and Van Ess J: Single-dose 
half-body irradiation for palliation of multiple bone metastases from solid tumors. Final radiation therapy oncology group report. Cancer 58(1): 29-36, 1986. PMID: 2423225. DOI: 10.1002/10970142(19860701)58:1<29::aid-cncr2820580107>3.0.co;2-2

11 Common terminology criteria for adverse events (ctcae) version 4.0., 2010. Available from: https://www.eortc.be/services/ doc/ctc/ctcae_4.03_2010-06-14_quickreference_5x7.pdf (last accessed September 11, 2019)

12 Dutz A and Lock S: Competing risks in survival data analysis. Radiother Oncol 130: 185-189, 2019. PMID: 30314718. DOI: 10.1016/j.radonc.2018.09.007

13 Tey J, Choo BA, Leong CN, Loy EY, Wong LC, Lim K, Lu JJ and Koh WY: Clinical outcome of palliative radiotherapy for locally advanced symptomatic gastric cancer in the modern era. Medicine (Baltimore) 93(22): e118, 2014. PMID:25396330. DOI: $10.1097 / \mathrm{MD} .0000000000000118$

14 Sagone AL Jr., Lawrence T and Balcerzak SP: Effect of smoking on tissue oxygen supply. Blood 41(6): 845-851, 1973. PMID: 4712210 .

15 Chen AM, Chen LM, Vaughan A, Sreeraman R, Farwell DG, Luu Q, Lau DH, Stuart K, Purdy JA and Vijayakumar S: Tobacco smoking during radiation therapy for head-and-neck cancer is associated with unfavorable outcome. Int $\mathbf{J}$ Radiat Oncol Biol Phys 79(2): 414-419, 2011. PMID: 20399030. DOI: 10.1016/j.ijrobp.2009.10.050

16 Foerster B, Pozo C, Abufaraj M, Mari A, Kimura S, D'Andrea D, John H and Shariat SF: Association of smoking status with recurrence, metastasis, and mortality among patients with localized prostate cancer undergoing prostatectomy or radiotherapy: A systematic review and meta-analysis. JAMA Oncol 4(7): 953-961, 2018. PMID: 29800115. DOI: 10.1001/ jamaoncol.2018.1071
17 Oken MM, Creech RH, Tormey DC, Horton J, Davis TE, McFadden ET and Carbone PP: Toxicity and response criteria of the eastern cooperative oncology group. Am J Clin Oncol 5(6): 649-655, 1982. PMID: 7165009.

18 Jang RW, Caraiscos VB, Swami N, Banerjee S, Mak E, Kaya E, Rodin G, Bryson J, Ridley JZ, Le LW and Zimmermann C: Simple prognostic model for patients with advanced cancer based on performance status. J Oncol Pract 10(5): e335-341, 2014. PMID: 25118208. DOI: 10.1200/JOP.2014.001457

19 Gooley TA, Leisenring W, Crowley J and Storer BE: Estimation of failure probabilities in the presence of competing risks: New representations of old estimators. Stat Med 18(6): 695-706, 1999. PMID: 10204198. DOI: 10.1002(sici)1097-0258(1999 90330)18;6<695::aid-sim60>3.0.co;2-o

$20 \mathrm{Kim}$ HT: Cumulative incidence in competing risks data and competing risks regression analysis. Clin Cancer Res 13(2 Pt 1): 559-565, 2007. PMID: 17255278. DOI: 10.1158/10780432.CCR-06-1210

21 Southern DA, Faris PD, Brant R, Galbraith PD, Norris CM, Knudtson ML, Ghali WA and Investigators A: Kaplan-meier methods yielded misleading results in competing risk scenarios. J Clin Epidemiol 59(10): 1110-1114, 2006. PMID: 16980152. DOI: $10.1016 /$ j.jclinepi.2006.07.002
Received August 6, 2019

Revised September 10, 2019

Accepted September 11, 2019 